\title{
Spatial measures between human poses for classification and understanding
}

Hauberg, Søren; Pedersen, Kim Steenstrup

Published in:

Articulated Motion and Deformable Objects

DOI:

10.1007/978-3-642-31567-1_3

Publication date:

2012

Document version

Early version, also known as pre-print

Citation for published version (APA):

Hauberg, S., \& Pedersen, K. S. (2012). Spatial measures between human poses for classification and understanding. In F. J. Perales, R. B. Fisher, \& T. B. Moeslund (Eds.), Articulated Motion and Deformable Objects: 7th International Conference, AMDO 2012, Port d'Andratx, Mallorca, Spain, July 11-13, 2012. Proceedings (pp. 26-36). Springer. Lecture notes in computer science Vol. 7378 https://doi.org/10.1007/978-3642-31567-1_3 


\title{
Spatial Measures between Human Poses for Classification and Understanding
}

\author{
Søren Hauberg and Kim Steenstrup Pedersen \\ 1 Max Planck Institute for Intelligent Systems, Perceiving Systems \\ Spermannstrasse, 72076 Tübingen, Germany \\ soren. hauberg@tue.mpg.de \\ 2 Dept. of Computer Science, University of Copenhagen \\ Universitetsparken 1, 2100 Copenhagen, Denmark \\ kimstp@diku.dk
}

\begin{abstract}
Statistical analysis of humans, their motion and their behaviour is a very well-studied problem. With the availability of accurate motion capture systems, it has become possible to use such analysis for animation, understanding, compression and tracking of human motion. At the core of the analysis lies a measure for determining the distance between two human poses; practically always, this measure is the Euclidean distance between joint angle vectors. Recent work [7] has shown that articulated tracking systems can be vastly improved by replacing the Euclidean distance in joint angle space with the geodesic distance in the space of joint positions. However, due to the focus on tracking, no algorithms have, so far, been presented for measuring these distances between human poses. In this paper, we present an algorithm for computing geodesics in the Riemannian space of joint positions, as well as a fast approximation that allows for large-scale analysis. In the experiments we show that this measure significantly outperforms the traditional measure in classification, clustering and dimensionality reduction tasks.
\end{abstract}

\section{Modelling Human Poses}

For many years researchers in different research fields have studied image sequences of moving humans and tried to reason about human behaviour. Examples of such reasoning include articulated tracking of the individual limbs in the human body [1,6,18,22], clustering to e.g. learn a discrete vocabulary of human motion (visual words) [5, 13], action recognition [25] and dimensionality reduction $[9,19,23]$. We also note a focus on statistical analysis of humans in animation [4,21,24] and robotics [17]. Due to the complexity of the human body it is common to use a rather simple body model consisting of only a few selected mass-less bones, which gives the kinematic skeleton [3]. This "stick figure" (see fig. 1a) is complex enough to capture many aspects of human behaviour, but simple enough to give tractable algorithms. In the kinematic skeleton, bone lengths are assumed constant such that the joint angles between connected bones constitute the only degrees of freedom in the model. It is, thus, common to learn models directly in the space of these joint angles, which can be treated as a Euclidean space. This is computationally efficient, but, as we shall later see, leads to an unnatural distance measure. Alternatively, we can model humans in the space of joint positions, which is more 
similar to what is actually observed in images of humans. However, the space of joint positions is a non-trivial Riemannian manifold [6], which complicates analysis. In this paper, we provide an algorithm for computing geodesics on this manifold as well as a very efficient approximation scheme. This allows us to perform larger studies, which show that changing the distance measure has profound impact on the performance of the learned models.

\subsection{Related Work}

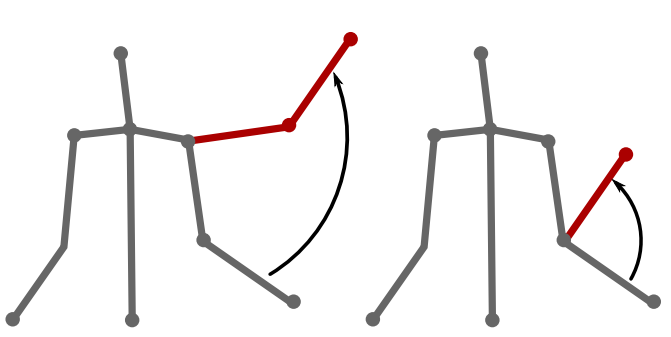

(a)

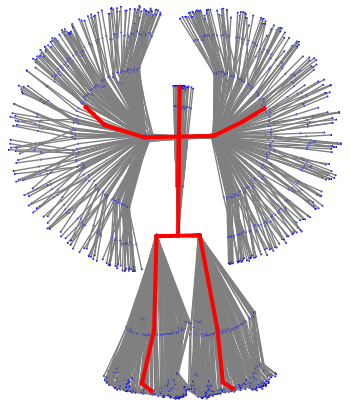

(b)

Fig. 1. Different distance measures. (a) Two motions of equal size $\left(90^{\circ}\right)$ under the joint angle metric. The example is adapted from [7]. (b) The mean pose and data from a jumping jack sequence under the Euclidean joint position distance measure. The resulting mean pose does not respect the skeleton structure of the data.

As previously stated, most of the work concerning analysis of human movement represents the human body with the kinematic skeleton. For a given skeleton, a human pose can be represented by a vector of all joint angles; this vector is often assumed to be Euclidean such that standard techniques apply. From the vector of joint angles, we can compute joint positions by recursively orienting each bone in the skeleton according to the angle vector. This process is known as forward kinematics [3]. From a geometric point of view, we can think of forward kinematics as recursively selecting a point on a 2 -sphere with centre corresponding to the previous bone end-point and radius corresponding to the bone length.

Letting $\theta$ denote the joint angles of the kinematic skeleton we define the joint angle metric as

$$
\operatorname{dist}_{\theta}\left(\theta_{1}, \theta_{2}\right)=\left\|\theta_{1}-\theta_{2}\right\|_{2},
$$

i.e. the Euclidean metric in joint angle space. This metric appears frequently in the literature $[4,9,12,17-19,22-24]$.

In articulated tracking, i.e. sequential pose estimation, the joint angle metric is often used as part of a Gaussian prior to ensure smooth motion estimates, see e.g. $[8,11,12$, 
18]. When learning activity specific priors, the angle metric is also used for comparing training data, e.g. for learning Gaussian Process Latent Variable Models [22-24] and for manifold learning techniques $[9,19]$. Furthermore, these learning schemes also often use the angle metric for regularisation purposes.

The angle metric is often used due to its simplicity, but, as pointed out by Hauberg et al. [7], it has little relation to the visual size of the motion. Fig. 1a shows two different motions that all have the same size under the joint angle metric, as only one joint angle has been changed 90 degrees. The motions, however, appear to have different size, with the motion on the left appearing larger than the motion on the right. This behaviour is due to the fact that the angle metric ignores the structure of the skeleton, i.e. the size of the bones as well as how they are connected to each other.

In robotics and computer vision it is common to use twists and exponential maps $[1,10]$ instead of joint angles. This has great practical impact on, e.g., optimisation, but the underlying distance measure still ignores bone lengths and connectivity. The same can be said for quaternion [3] representations.

An alternative metric was suggested by Tournier et al. [21] for the purpose of motion compression. This measure treats the kinematic skeleton as a product of one sphere per bone, such that the metric is the sum of distances on $S O(3)$. This measure incorporates knowledge of bone lengths and has a simple physical interpretation as it works in the world coordinate system. The measure, however, ignores how bones are connected, i.e. it cannot capture that moving the upper arm causes the lower arm to move as well. A better metric should incorporate both knowledge of bone lengths and bone connectivity.

\section{The Space of Joint Positions}

The simplest possible distance measure that takes both bone lengths and connectivity into account is the Euclidean distance in the space of joint positions. This measure is simple, easy to interpret and fast to compute. It can, however, be a problematic measure when used for statistics; consider the jumping jacks sequence data shown in fig. $1 \mathrm{~b}$. In the figure we also show the mean pose computed using the Euclidean joint position distance and as can be seen the limbs of the mean pose are substantially smaller than those found in the original data. Clearly, the choice of distance measure has resulted in statistics that fail to preserve the skeleton structure.

The immediate solution is to perform analysis in the Euclidean space, but with the restriction that only parts of space corresponding to a valid skeleton configuration is allowed. The kinematic manifold [6] is exactly this part of the Euclidean space. The manifold $\mathcal{M}$ is defined as the space of all possible joint positions,

$$
\mathcal{M}=\{F(\theta) \mid \theta \in \Theta\},
$$

where $F$ is the forward kinematics function, i.e. the function that maps from joint angles to joint positions, and $\Theta$ is the set of legal joint angles, i.e. it encodes joint constraints. This is a Riemannian manifold embedded in $\mathbb{R}^{3 L}$, where $L$ is the number of joints in the skeleton [6]. As such, $F(\theta)$ is simply the vector containing the position of all joints at the pose $\theta$. The manifold structure arises due to the constraint that connected joints have a fixed distance between them. This means that any point on $\mathcal{M}$ will be a valid 
skeleton, i.e. it will respect both bone lengths and joint constraints. If we confine our statistics to this manifold, we avoid the problems of working directly in the Euclidean space of joint positions.

As discussed in [7], the geodesic distance on $\mathcal{M}$ between two poses $\theta_{0}$ and $\theta_{N}$ is the visually natural measure of the length of the spatial curves $c$ that joints move along, i.e.

$$
\operatorname{dist}_{\mathcal{M}}\left(\theta_{0}, \theta_{N}\right)=\min _{\begin{array}{c}
c(\tau) \in \mathcal{M}, \\
c(0)=F\left(\theta_{0}\right) \\
c(1)=F\left(\theta_{N}\right)
\end{array}} \mathcal{L}(c)
$$

where $\mathcal{L}$ denotes the length of a curve $c$, i.e.

$$
\mathcal{L}(c)=\int_{0}^{1}\left\|\frac{\partial c(\tau)}{\partial \tau}\right\| \mathrm{d} \tau
$$

As the geodesic curve $c$ is restricted to $\mathcal{M}$ any point on the curve is a valid skeleton, which makes the measure different from the ordinary Euclidean measure in joint position space.

While the distance measure in eq. 3 provides a natural notion of the "size" of a movement between two poses, no practical algorithms have yet been provided for computing this distance and the corresponding geodesics.

\subsection{Computing Geodesics and Distances}

In order to compute geodesics, and hence distances, between two poses, we need to minimise the integral in eq. 4 . This can be vastly simplied by utilising that extremal points of eq. 3 coincides with extremal points of the curve energy [2],

$$
\mathcal{E}(c)=\int_{0}^{1}\left\|\frac{\partial c(\tau)}{\partial \tau}\right\|^{2} \mathrm{~d} \tau .
$$

To compute a geodesic, we can, thus, iteratively evolve an initial curve $c$ towards a curve minimizing eq. 5 . To do this, we discretise a given initial curve $c$ connecting $\theta_{0}$ and $\theta_{N}$ by sampling $N-1$ points between $\theta_{0}$ and $\theta_{N}$. The curve is, thus, approximated by $\hat{c}=\left\{\theta_{0}: \theta_{N}\right\}=\left\{\theta_{0}, \ldots, \theta_{N}\right\}$. We can then compute the energy of this discrete curve as

$$
\mathcal{E}\left(\theta_{0}: \theta_{N}\right) \approx \sum_{n=1}^{N}\left\|F\left(\theta_{n-1}\right)-F\left(\theta_{n}\right)\right\|^{2},
$$

which is a straight-forward discretisation of the integral in eq. 5. In order to minimise eq. 6 we then seek the set of intermediate points $\theta_{1}: \theta_{N-1}$ that minimises $\mathcal{E}$.

The derivative of the approximate curve energy can be computed in closed-form as (derived in appendix A)

$$
\frac{\partial \mathcal{E}}{\partial \theta_{i}}=2\left(F\left(\theta_{i-1}\right)-F\left(\theta_{i+1}\right)^{T} \mathbf{J}_{\theta_{i}}\right.
$$

where $\mathbf{J}_{\theta_{i}}=\frac{\partial F}{\partial \theta_{i}}$ is the Jacobian of $F$ evaluated in $\theta_{i}$, which can easily be computed in closed-form, cf. [26]. With this, we perform minimisation using a trust-region gradient descent algorithm. 


\subsection{Approximations and the Geometry of the Kinematic Manifold}

One problem with the presented algorithm for computing geodesics is that it can be rather time consuming due to its iterative nature. In most statistical computations we need to repeatedly calculate distances, so this quickly becomes a bottleneck. We, thus, look for fast alternatives that do not rely on an iterative optimisation. For this we take a closer look at the geometry of the kinematic manifold.

The strength of the joint angle metric is that it is just the Euclidean metric in joint angle space, which makes it fast and easy to compute. As discussed above, and as will be illustrated by our experiments, this metric does, however, not model human pose data well. Another issue with this metric is that it is dependent on the choice of root node in the kinematic skeleton, whereas the joint position metric is not.

Our approximation of the geodesic curve on the kinematic manifold utilizes the simplicity of computing the joint angle metric for a particular choice of root node in the kinematic skeleton. As we shall see, this choice turns out to be a good approximation for the geodesic curve in joint position space, even though the corresponding distances - joint angle distance and joint position distance - are vastly different. This choice of approximation rests on the following geometric considerations.

The simplest case of a kinematic manifold is when the skeleton only has one bone. In this case the kinematic manifold reduces to the well-known 2 -sphere in $\mathbb{R}^{3}$, as is illustrated in the left of fig. 2. When the sphere is parametrised using angles, it is wellknown that geodesics in the joint position metric will form straight lines in the angle space.
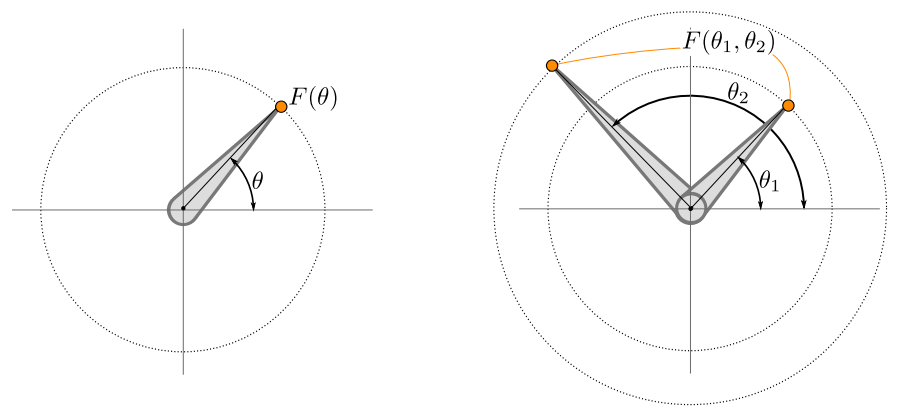

Fig. 2. Illustrations of the kinematic manifold for simple skeletons. Left: for skeletons with only one bone, the kinematic manifold is a sphere, where the centre coincides with the root of the skeleton. Right: for skeletons with two bones we can place the root of the skeleton at the joint connecting the two bones. The kinematic manifold then corresponds to the product of two spheres with a shared centre.

The situation is similarly simple when the skeleton has two bones. If we pick the root of the skeleton as the joint that connects the two bones, then the kinematic manifold is the product of two spheres with a shared centre that coincides with the root, see the 
right of fig. 2. If we consider geodesics in the joint position metric, then it is again clear that it will consist of straight lines in the joint angle space representing the two spheres.

These observations provide us with the hint that many geodesics in the joint position metric form straight lines in joint angle space. As the kinematic manifold is Riemannian, we know that the metric tensor varies smoothly, such that we most often will see smooth changes in geodesics when the end-points move. Hence, we will use the straight line in joint angle space connecting the end-points as an approximation of the geodesic, i.e. $c_{\text {line }}=\left\{\theta_{0}, \frac{1}{N} \theta_{0}+\frac{N-1}{N} \theta_{N}, \ldots, \theta_{N}\right\}$. This path is a geodesic under the joint angle metric and the $S O(3)$ product metric suggested by Tournier et al. [21], but the actual geodesic lengths differ. It is worth noting that when using the length of non-geodesic curves as a distance measure, we are not guaranteed that the measure satisfies the triangle inequality. In many practical scenarios this is, however, acceptable; see e.g. the work on metric learning by Ramanan and Baker [14] where straight lines are also used to approximate geodesics.

As we shall see in sec. 3.1, the suggested curve turns out to be a very good approximation of the true geodesic. As this approximation does not require any iterative optimisation it can be computed very fast, which allows for large scale experiments.

\section{Experiments}

The purpose of the experiments is to show that the approximate spatial geodesic distance improves different machine learning tasks, such as dimensionality reduction, clustering and classification. Here we focus on rather simple techniques as these are most well-understood, allowing us to easier analyse the impact of changing the distance measure. We will use data collected with an optical motion capture system as large datasets with different people and activities are readily available online ${ }^{3}$.

In all experiments we only consider the human pose and disregard global position and orientation.

\subsection{Quality of Geodesic Approximation}

Our first experiment is concerned with how well the approximate geodesic curve, described in sec. 2.2, approximates the true geodesic. This turns out to be a good approximation of the true geodesic, but it is much faster to compute.

As a first qualitative experiment we pick two random poses from a walking sequence, form the approximate geodesic curve $c_{\text {line }}$ as described in sec. 2.2, and compute the length minimising geodesic curve $c_{\text {geodesic }}$ using the approach described in sec. 2.1. We discretise the curves using 10 sample points and compute the first two principal components of the discrete points, which allows us to visualise the curves, cf. fig. 3a. In the figure, the background is colour coded with the trace of the local metric tensor, which can be considered a measure of the local curvature of $\mathcal{M}$. The expression for the metric tensor is derived in appendix B. We visually see that the approximate geodesic curve is very similar to the length-minimising geodesic.

\footnotetext{
${ }^{3}$ We use the Carnegie Mellon dataset from http://mocap.cs.cmu.edu/.
} 


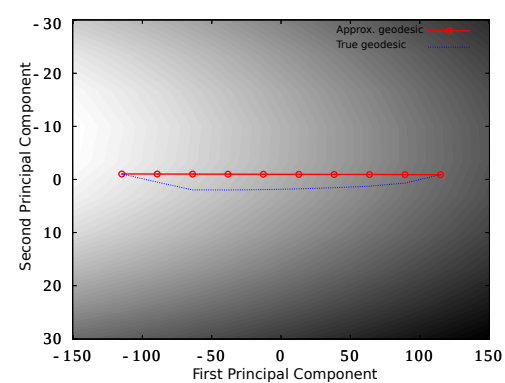

(a)

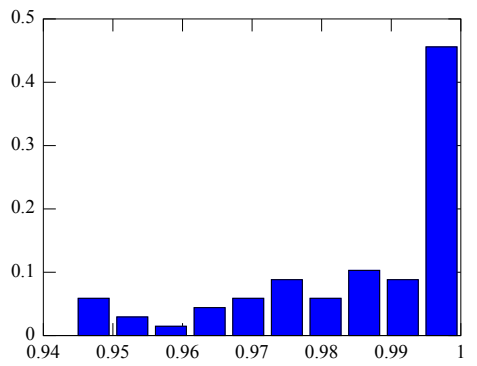

(b)

Fig. 3. (a) A visualisation of the geodesic path and the straight-line approximation. The coordinate system are the first two principal components of the sample points along the curves expressed in joint angles. Notice the size of the $y$-axis compared to the $x$-axis; this is an indication that the two curves are very similar. The background colouring of each point is proportional to the trace of the local metric tensor expressed in joint angles, i.e. a measure of the local curvature of $\mathcal{M}$. (b) A histogram over the ratio in eq. 8. Notice that the smallest observed value of this ratio is 0.94 , which tells us that the approximation is quite good.

Next, we seek to quantify the above observations. Given an approximate geodesic and a length minimising geodesic between two poses, we can measure how much they differ as

$$
r=\frac{\mathcal{L}\left(c_{\text {geodesic }}\right)}{\mathcal{L}\left(c_{\text {line }}\right)}
$$

As the length of the true geodesic is shorter than or equal to the length of the approximate curve $r \in[0,1]$. In fig. 3b we show a histogram of the ratios for 100 random pairs of poses. As can be seen, the ratio is always very close to 1, and the lowest observed ratio is 0.94 , which tells us that the approximation curve is indeed quite good. In practice, the true geodesic distance is too expensive to compute in large studies, so we only consider the approximation in the remaining parts of the paper.

\subsection{Dimensionality Reduction}

One of the most basic machine learning tasks is to learn a low-dimensional model from high-dimensional data. The most basic model for such dimensionality reduction tasks is principal component analysis, which can be generalised to metric spaces, such as Riemannian manifolds, with multidimensional scaling (MDS) [15]. This algorithm finds the Euclidean representation of a set of points that preserve pair-wise distances of the original data as well as possible.

As a first qualitative experiment we consider two sequences corresponding to the walk cycles of two people. We then compute the first two principal components of this data according to the angular metric and the geodesic approximation of the joint position metric. In fig. $4 \mathrm{a}$ we show the results for the angular metric. As can be seen, the 
learned model is essentially bimodal, where each mode corresponds to the two different persons. As such, the model fails to capture any similarity between the walking styles, i.e. we cannot expect this model to generalise to new people. In fig. $4 \mathrm{~b}$ we show the comparable results for the geodesic approximation of the joint position metric. We see that the resulting model is essentially unimodal, which indicates that the learned model has captured the similarities between the two different walking styles.

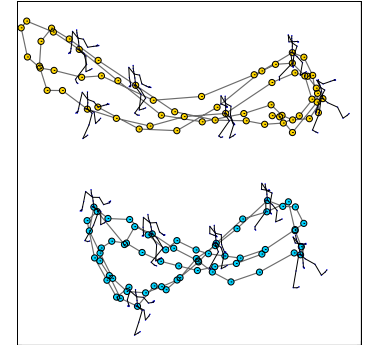

(a)

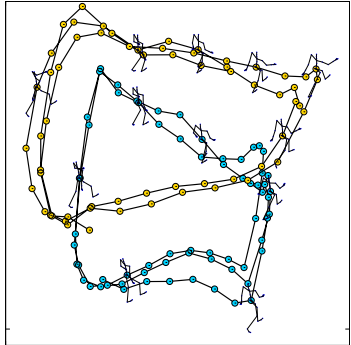

(b)

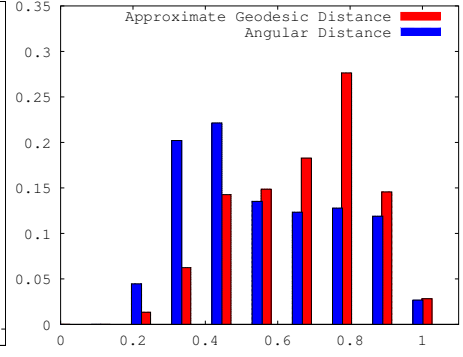

(c)

Fig. 4. (a)-(b) Illustration of results attained for the different metrics when using multidimensional scaling for dimensionality reduction; (a) show results for the angular metric and (b) the spatial distance. The colours indicate different persons. Note how the angular metric fails to capture any similarity between the different walking styles. (c) A histogram of the percentage of data explained by the first principal component under the two different metrics. The approximate geodesic distance significantly outperforms the angular distance measure according to a MannWhitney $U$-test. These figures are best viewed in colour.

In order to quantify the above observations, we measure how large a percentage of the data is captured by the first principal component under the different distance measures. We do this for 673 independent sequences and show a histogram of these percentages in fig. 4c. For the results under the joint angle metric we see a mode of explaining $40 \%$ of the data, whereas the mode is at $80 \%$ under the approximate joint position geodesic distance. Using a Mann-Whitney $U$-test we see that the approximate joint position geodesic distance model significantly explains more of the data in the first component than the angular distance model ( $p$-value below $10^{-6}$ ). This is a strong indication that the approximate geodesic distance is better for dimensionality reduction than the angular distance measure. It is worth noting that we have performed similar experiments with other dimensionality reduction techniques, such as Isomap [20], and see similar behaviour.

\subsection{Classification}

Another classical machine learning task is that of classification. To illustrate the impact of the choice of distance measure, we pick a simple two-class problem consisting of distinguishing walking from running. As we use motion capture data, we can expect little noise, so we expect this problem to be easily solved. We randomly select 10 
walking and 10 running sequences, and, in a leave-one-out fashion, test on 1 walking sequence and train on the 19 remaining. We down-sample the sequences to $12 \mathrm{~Hz}$ such that we in total use 1096 data points. We choose to use the nearest neighbour classifier to maximum stress on the choice of metric.

For this trivial classification problem, the approximate geodesic distance achieves $100 \%$ success rate, which clearly illustrates that the problem is not particularly hard. The angular metric, on the other hand, only achieves a $90 \%$ success rate, which is a very disappointing result that shows the limits of the joint angle metric.

\subsection{Clustering}

The last classical machine learning task we consider is that of clustering. Again, the choice of distance measure can potentially have a large impact on the results attained as this is often what determines whether two data points belong to the same cluster. We pick a simple clustering technique that introduces little extra knowledge other than what is provided by the metric. Specifically, we use the medioid-shift algorithm [16] with a Gaussian kernel, where the bandwidth is selected to achieve the correct number of clusters. We use the same data as in the classification example, i.e. 10 walking sequences and 10 running sequences. We combine all pairs of sequences from different classes, which gives us 100 clustering tasks with two clusters. We then measure the percentage of data points assigned to the correct class and report the average. When using the angle metric we assign $60 \%$ of the data to the correct class and $65 \%$ when using the approximate joint position geodesic distance, which is a small but noticeable improvement.

\section{Discussion}

In this paper, we have provided a novel algorithm for computing geodesics on the kinematic manifold for representing joint positions in human skeleton models. This provides a mathematically well-founded and visually natural distance measure for comparing human poses. This algorithm, however, depends on iterative optimisation, which can make it impractical for large studies. For this reason, we have also provided a fast and accurate algorithm for approximating the geodesics. In our experiments this approximation only differs slightly from the true geodesic. More thorough experiements in this direction would, however, be interesting.

In the experimental part of the paper, we perform a number of comparisons of the new metric with the standard joint angle measure, which is used practically everywhere else in the literature. The experiments included the classical machine learning tasks clustering, classification and dimensionality reduction. In all cases we saw improved results. Sometimes the improvements are drastic, as in the case of dimensionality reduction, and other times the improvements are minor, as in the case of clustering. However, in general we see improvements in all experiments. As the suggested measure can be computed efficiently, we see little reason to use the standard joint angle measure in the future.

Acknowledgements: S.H. would like to thank the Villum Foundation for financial support. 


\section{References}

1. Bregler, C., Malik, J., Pullen, K.: Twist based acquisition and tracking of animal and human kinematics. International Journal of Computer Vision 56 (February 2004) 179-194

2. Carmo, M.P.D.: Differential Geometry of Curves and Surfaces. Prentice Hall (1976)

3. Erleben, K., Sporring, J., Henriksen, K., Dohlmann, H.: Physics Based Animation. Charles River Media (August 2005)

4. Grochow, K., Martin, S.L., Hertzmann, A., Popović, Z.: Style-based inverse kinematics. ACM Transaction on Graphics 23(3) (2004) 522-531

5. Guerra-Filho, G., Aloimonos, Y.: A language for human action. Computer 40 (2007) 42-51

6. Hauberg, S., Sommer, S., Pedersen, K.S.: Gaussian-like Spatial Priors for Articulated Tracking. In: ECCV. Volume 6311 of LNCS., Springer (2010) 425-437

7. Hauberg, S., Sommer, S., Pedersen, K.S.: Natural metrics and least-committed priors for articulated tracking. Image and Vision Computing (2011)

8. Kjellström, H., Kragić, D., Black, M.J.: Tracking people interacting with objects. In: IEEE CVPR. (2010)

9. Lu, Z., Carreira-Perpinan, M., Sminchisescu, C.: People Tracking with the Laplacian Eigenmaps Latent Variable Model. In: NIPS 20. MIT Press (2008) 1705-1712

10. Murray, R.M., Li, Z., Sastry, S.S.: A Mathematical Introduction to Robotic Manipulation. CRC Press (March 1994)

11. Poon, E., Fleet, D.J.: Hybrid monte carlo filtering: Edge-based people tracking. IEEE Workshop on Motion and Video Computing 0 (2002) 151

12. Poppe, R.: Vision-based human motion analysis: An overview. Computer Vision and Image Understanding 108(1-2) (2007) 4-18

13. Priyamvada, K.K., Kahol, K., Tripathi, P., Panchanathan, S.: Automated gesture segmentation from dance sequences. In: Int. Conf. on Automatic Face and Gesture Recognition. (2004)

14. Ramanan, D., Baker, S.: Local distance functions: a taxonomy, new algorithms, and an evaluation. TPAMI (4) (2011)

15. Ripley, B.D.: Pattern recognition and neural networks. Cambridge University Press (1996)

16. Sheikh, Y.A., Khan, E.A., Kanade, T.: Mode-seeking by medoidshifts. In: IEEE 11th International Conference on Computer Vision. (2007) 1-8

17. Shon, A.P., Grochow, K., Rao, R.P.: Robotic imitation from human motion capture using Gaussian processes. (2005)

18. Sidenbladh, H., Black, M.J., Fleet, D.J.: Stochastic tracking of $3 \mathrm{~d}$ human figures using $2 \mathrm{~d}$ image motion. In: ECCV. Volume II of LNCS 1843., Springer (2000) 702-718

19. Sminchisescu, C., Jepson, A.: Generative modeling for continuous non-linearly embedded visual inference. In: ICML ’04, ACM (2004) 759-766

20. Tenenbaum, J.B., Silva, V., Langfor, J.C.: A global geometric framework for nonlinear dimensionality reduction. Science 290(5500) (December 2000) 2319-2323

21. Tournier, M., Wu, X., Courty, N., Arnaud, E., Reveret, L.: Motion compression using principal geodesics analysis. Computer Graphics Forum 28(2) (2009) 355-364

22. Urtasun, R., Fleet, D.J., Fua, P.: 3D People Tracking with Gaussian Process Dynamical Models. In: IEEE CVPR. (2006) 238-245

23. Urtasun, R., Fleet, D.J., Hertzmann, A., Fua, P.: Priors for people tracking from small training sets. In: ICCV. Volume 1. (2005) 403-410

24. Wang, J.M., Fleet, D.J., Hertzmann, A.: Gaussian Process Dynamical Models for Human Motion. IEEE PAMI 30(2) (2008) 283-298

25. Yao, A., Gal, J., Fanelli, G., van Gool, L.: Does human action recognition benefit from pose estimation? In: BMVC. (2011)

26. Zhao, J., Badler, N.I.: Inverse kinematics positioning using nonlinear programming for highly articulated figures. ACM Transaction on Graphics 13(4) (1994) 313-336 


\section{A Derivative of Curve Energy}

We remind the reader that the energy of a curve on $\mathcal{M}$ is approximated as

$$
\mathcal{E}\left(\theta_{0}: \theta_{N}\right) \approx \sum_{n=1}^{N}\left\|F\left(\theta_{n-1}\right)-F\left(\theta_{n}\right)\right\|^{2}
$$

As this function only depends on $\theta_{1}: \theta_{N-1}$, the gradient can be written as

$$
\nabla \mathcal{E}=\left(\begin{array}{c}
\frac{\partial \mathcal{E}}{\partial \theta_{1}} \\
\vdots \\
\frac{\partial \mathcal{E}}{\partial \theta_{N-1}}
\end{array}\right)
$$

Each of these partial derivatives can easily be computed using the chain rule, which gives us

$$
\begin{aligned}
\frac{\partial \mathcal{E}}{\partial \theta_{i}} & =\frac{\partial}{\partial \theta_{i}}\left\|F\left(\theta_{i-1}\right)-F\left(\theta_{i}\right)\right\|^{2}+\frac{\partial}{\partial \theta_{i}}\left\|F\left(\theta_{i}\right)-F\left(\theta_{i+1}\right)\right\|^{2} \\
& =2\left(\left(F\left(\theta_{i-1}\right)-F\left(\theta_{i}\right)\right)+\left(F\left(\theta_{i}\right)-F\left(\theta_{i+1}\right)\right)^{T} \mathbf{J}_{\theta_{i}}\right. \\
& =2\left(F\left(\theta_{i-1}\right)-F\left(\theta_{i+1}\right)^{T} \mathbf{J}_{\theta_{i}},\right.
\end{aligned}
$$

where $\mathbf{J}_{\theta_{i}}=\frac{\partial F}{\partial \theta_{i}}$ is the Jacobian of $F$ evaluated in $\theta_{i}$. This can easily be computed in closed-form, cf. [26].

\section{B The Metric Tensor of $\mathcal{M}$ in Angle Space}

The background colour of fig. 3a is the trace of the metric tensor of $\mathcal{M}$ expressed in the joint angle space. The metric tensor is in itself an interesting object so we derive it here.

The length of a curve $c(t):[0,1] \rightarrow \Theta$ measured on $\mathcal{M}$ is defined as

$$
\begin{aligned}
\mathcal{L}(c) & =\int_{0}^{1}\left\|\frac{\partial F(c(t))}{\partial t}\right\| \mathrm{d} t=\int_{0}^{1}\left\|\frac{\partial F(c(t))}{\partial c(t)} \frac{\partial c(t)}{\partial t}\right\| \mathrm{d} t \\
& =\int_{0}^{1}\left\|\mathbf{J}_{c(t)} c^{\prime}(t)\right\| \mathrm{d} t=\int_{0}^{1} \sqrt{c^{\prime}(t)^{T} \mathbf{J}_{c(t)}^{T} \mathbf{J}_{c(t)} c^{\prime}(t)} \mathrm{d} t,
\end{aligned}
$$

where $\mathbf{J}_{c(t)}$ is the Jacobian of $F$ evaluated at $c(t)$ and $c^{\prime}(t)$ is the derivative of $c$ with respect to $t$. Eq. 15 tells us that the metric tensor of $\mathcal{M}$ at a point $\theta$ in joint angle space is given by $\mathbf{J}_{\theta}^{T} \mathbf{J}_{\theta}$. 